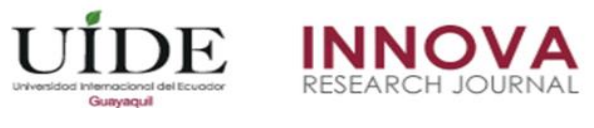

INNOVA Research Journal, ISSN 2477-9024

(Febrero, 2017). Vol. 2, No.2 pp. 55-61.

DOI: https://doi.org/10.33890/innova.v2.n2.2017.121

URL: http://revistas.uide.edu.ec/index.php/innova/index

Correo: innova@uide.edu.ec

\title{
La política de protección de tierras para la población desplazada en Colombia entre 1997.2014: análisis de caso de una etnografía del estado
}

\section{The politics of land protection for the displaced population in Colombia between 1997.2014: case analysis of a state ethnography}

Nadia Margarita Rodríguez J.

Universidad Internacional SEK, Ecuador

Autor para correspondencia: nadia.rodriguez@uisek.edu.ec

Fecha de recepción: 24 de Noviembre de 2016 - Fecha de aceptación: 10 de enero de 2017

Resumen: En este artículo reflexiona sobre los marcos teórico-metodológicos y los aportes de una investigación realizada sobre la política de protección de los bienes de la población desplazada y su transformación en política de restitución de tierras. Esta investigación implicó, entre otros abordajes, una etnografía institucional en el Proyecto de Protección de Tierras para la Población Desplazada (PPTP en adelante). El estudio resultó ser un caso etnográfico bastante particular; por una parte por las características del PPTP que se salen de la estructura normal de funcionamiento de una institución pública, y por otra parte porque durante la realización de la investigación se produjo un viraje significativo en las lógicas de gobierno que dieron origen a la transformación del PPTP en la Unidad Administrativa Especial para la Restitución de Tierras de la Población Desplazada (URT en adelante). En este sentido se discutirá sobre tres aspectos: por una parte sobre la pertinencia de los marcos teóricos utilizados para analizar lo político y al Estado (Trouillout 2003, 2011, Rose y Millar 1992, Sharma y Guptha 2006); por otra parte los retos metodológicos de esta aproximación etnográfica en un contexto político cambiante y finalmente aportaremos algunas reflexiones sobre los alcances de la investigación aplicada en facetas extra académicas.

Palabras Clave: Etnografía de lo político; protección de tierras; investigación aplicada

Abstract: The paper presents the theoretical and methodological frameworks and the contributions of a research on the protection of property policy for displaced population, and its transformation into land restitution policy. This research involved, among other approaches, institutional ethnography in the Land Protection Project for the Displaced Population (PPTP). The study was a very particular ethnographic case, because the operating procedure of the PPTP was not conventional for an state institution, and also because during the research there was a significant change in the logic of government that led to the transformation of PPTP into an Special Administrative Unit for the Land Restitution of the Displaced Population (URT). In this sense, the paper will discuss three aspects: The relevance of the theoretical frameworks used to analyze the political and the State (Trouillout 2003, 2011, Rose and Miller 1992, Sharma and Guptha 2006); the methodological challenges of this ethnographic approach in a changing political context; and finally, it will bring some reflections of applied research.

Keywords: Ethnography of politics; land protection; applied research 


\section{Introducción}

El objetivo de este documento es reflexionar sobre la puesta en marcha de la política de protección de los bienes de la población desplazada y su posterior transformación en política de restitución de tierras. Esta propuesta se inscribe en una investigación realizada entre el 2011 y el 2014, en la Escuela de Ciencias Humanas de la Universidad del Rosario, de Colombia.

La presentación de estos resultados busca poner en discusión los marcos conceptuales y metodológicos utilizados, al tiempo que intenta contribuir a la reflexión sobre la diversidad de espacios y la validez de la investigación antropológica en esferas extra académicas.

\section{Propuesta para una Etnografía del Estado y de la Política: El Caso de la Protección de Tierras para la Población Desplazada.}

La protección patrimonial de los bienes abandonados por la población víctima del desplazamiento forzado en Colombia fue enunciada por primera vez en el artículo 19 de la ley 387 de 1997. Para entonces, el fenómeno ${ }^{1}$ del desplazamiento había tomado dimensiones insospechadas, denunciadas en distintos ámbitos por organismos defensores de derechos humanos (Condhes 2009, 2011, Uprimny y Sanin 2006). La política para el desplazamiento atendió a la población, sin atacar el fenómeno ; y la protección de las tierras abandonadas solo se puso en marcha en el 2003 con la creación del "Proyecto de Protección de Tierras para la Población Desplazada"(en adelante PPTP), en donde se llevó a cabo esta etnografía. El proceso de implementación de la política de protección estuvo marcado por la interacción de múltiples fuerzas políticas, estrategias, e incluso algunas coincidencias, que hicieron posible la existencia y permanencia del proyecto y que hacen el caso interesante para esta reflexión. En 2011 con la Ley 1448 o Ley de víctimas, el camino de la protección abre paso a la restitución; está coyuntura se vivió al tiempo que se desarrollaba esta investigación e implicó retos interesantes en el análisis de las estructuras políticas y las lógicas de gobierno que eran objeto de la observación. Esta coyuntura también permitió ampliar el conocimiento sobre el tema y que se abriera oportunidades de intervención, tanto en una consultoría, como al interior de la Unidad especial de gestión de la restitución de tierras (URT en adelante).

Esta investigación teórica y metodológicamente partió de entender "lo político" como un campo con diversos intereses, actores y estrategias; y al Estado, como un aparato no coherente ni unificado (Swartz \& all 1966, Joseph y Nugent 1994, Haidar 2005). Partiendo de tres supuestos de M-R, Trouillot (2001, 2011): 1. El Estado más que un aparato es un conjunto de procesos, 2. El poder del Estado no está anclado en las instituciones y 3. Este poder se desarrolla en esferas que pueden enmarcarse en un espacio políticos externos. Al preguntar por el devenir de la política de protección se usó también la noción de Foucault de gubernamentalidad, para entender las diferentes lógicas de gobierno y poder que permitieron la implementación de la política. Dentro de esta perspectiva foucoultiana se identificaron las lógicas de gubernamentalidad de diversos actores, analizando intereses, jerarquías e interacciones incluso al interior del Estado (Rose y Miller 1992). Al igual que en la propuesta de Rose y Miller para analizar al Estado, se mostró que el poder político se ejerce a través de "un número de alianzas cambiantes entre las

\footnotetext{
${ }^{1}$ Varios autores han señalado las limitaciones de la política de desplazamiento forzado (Sarmiento 2000, Ibáñez y Vélez 2003, Villa 2006)
} 
autoridades de diversos proyectos" (Rose y Miller 1992:174). Estas alianzas constituyen tácticas en el ejercicio del poder que muchas veces determinan y sobrepasan al Estado.

Por otra parte, siguiendo la tradición de los estudios antropológicos del Estado, la etnografía privilegió el análisis de las prácticas de las personas, tanto de los usuarios, como de los funcionarios al interior del aparato burocrático (Sharma y Gupta 2006:10-18). Como lo demostró J Scott (1998), “el análisis de los procedimientos aparentemente rutinarios de los burócratas, proporciona importantes elementos para la comprensión de la micropolítica de trabajo estatal" (Sharma y Gupta 2006:11). J Ferguson (1994), por su parte, mostró cómo los funcionarios finalmente tienen un margen de ejercicio del poder en el gobierno, en el cual sus criterios, interpretaciones e interés crean formas particulares de aplicación de lo político.

Otro elemento interesante para entender esta política tiene que ver con la interacción de otros actores no gubernamentales, como las ONGs, la cooperación internacional y las organizaciones sociales. En este sentido, la investigación indagó sobre "las implicaciones y la influencia de estas organizaciones frente al Estado y la nación por una parte, y el Estado y la gobernabilidad en el otro" (Gupta 2006: 26).

Finalmente, la investigación analizó las diferentes fuerzas que tienen influencia en la política de protección, mostrando cómo el Estado es superado en el ejercicio del poder, tanto desde dentro (por los actores locales y las burocracias), cómo desde fuera (por organismos privados e internacionales) (Trouillot 2011, Gupta 2006).

Metodológicamente se hizo una etnografía al interior del PPTP y durante la transición para convertirse en la Unidad de Restitución de Tierras. Para ello, se revisaron más de 40 publicaciones del proyecto; se reconstruyó un archivo con más de 100 documentos jurídicos; se realizaron 35 entrevistas a funcionarios, magistrados, miembros de las organizaciones de víctimas, de Ongs y de organismos internacionales de cooperación; y también se realizaron observaciones en las oficinas de Bogotá, Cartagena y Carmen de Bolívar. El análisis etnográfico se centró en tres variables: i) La reconstrucción de los avances técnicos producidos en el PPTP, así como de los logros y resultados de la protección. ii) Se estudiaron los avances jurídicos producidos, y la interacción con las cortes y iii) Finalmente, se realizó un análisis del trabajo cotidiano de los funcionarios, indagando por sus percepciones sobre la puesta en marcha de la política de protección. Para esta presentación se profundizará sobre los retos metodológicos de realizar esta etnografía en un contexto convulso y de transformación de las lógicas gubernamentales.

\section{Los Retos de la Etnografía en Medio de la Transición Política}

Los hallazgos de la investigación evidencian las diferentes estrategias que utilizó el PPTP; estas fueron: mantener un bajo perfil, ciñéndose a la normativa y fortaleciendo un trabajo muy técnico y con una presencia constante en los lugares más afectados; todo ello permitió que el Proyecto mantuviera un margen de maniobra y de poder frente a la política. Paralelamente, la condición de financiarse con recursos externos, a la vez que les protegía, determinó una forma de funcionamiento más cercana a la de un organismo no gubernamental, lo cual no solo favoreció sus formas de funcionamiento, sino que le permitió mantenerse al margen de la manipulación 
política. Finalmente, la decisión de los financiadores de apoyar los procesos; de los funcionarios de ir más allá de sus propósitos misionales; así como el constante intercambio con las cortes, otras instituciones y Ongs defensoras de las víctimas, configuraron un mapa de relaciones y tácticas que no solamente produjo resultados en materia de protección, sino que facilitó el camino que puso en marcha la restitución.

Todo esto ocurre en un entorno político desfavorable, lo cual permitió mostrar cómo los mecanismos legales son un producto de varias lógicas de gubernamentalidad que se superponen. El caso evidencia cómo las decisiones políticas sobrepasan las instituciones públicas y muestra que detrás de las estrategias de cada actor hay intereses particulares, ya sean personales (de los funcionarios y activistas), locales (de las instituciones, las ONGs, las organizaciones de víctimas) o globales (de los organismos financiadores y de las cortes internacionales). La configuración de intereses de múltiples actores pasa por encima del hecho que no existiera voluntad política y, en un aspecto más amplio, refleja la incapacidad del poder político del Estado de mantener una unión, una coherencia entre las prácticas de sus instituciones (Abrams, 1988). En últimas, en el sentido de Sharma y Gupta, el Estado empieza a estar conformado por una "estructura híbrida que trasciende los límites entre arenas estatales y no estatales" (Sharma y Gupta 2006: 16).

Estos son, a muy grandes rasgos, los resultados de la investigación ${ }^{2}$, que pretendía llegar hasta la formulación de la Ley 1448. Pero la ley y particularmente su capítulo sobre restitución de tierras cambiaron por completo el panorama del PPTP, transformándolo en URT. De esta manera, mientras hacíamos el trabajo de campo para reconstruir la historia del PPTP, este iba desapareciendo y transformándose en un enorme aparato institucional (de 159 funcionarios pasaron a más de 1500 en cuestión de dos años). Lo interesante de este ejercicio fue asistir el proceso por el cual el Estado institucionaliza una política y vivir en carne propia, los agites, intrigas y decepciones que implicó esta transformación.

Por una parte, este proceso nos tomó por sorpresa; empezamos a trabajar el tema en el 2010 por el interés de una estudiante que quería hacer su tesis sobre algo relacionado con el PPTP. Preparamos la propuesta y cuando estaba lista sale la Ley 1448; esto implicó un revuelo importante y de la noche a la mañana el tema que estábamos trabajando se convirtió en titular de todas las noticias. Empezamos nuestras primeras aproximaciones al proyecto, justo a finales del 2011, cuando en el PPTP estaban corriendo para redactar los decretos reglamentarios para la restitución. Nuestras primeras entrevistas en 2012 las realizamos en pleno cambio viendo todo el movimiento; funcionarios que salían, otros que volvían y el estupor y trabajo desmedido de un grupo de personas que le pusieron el cuerpo y el alma a este proceso y que quedo registrado a lo largo de nuestras grabaciones. Ya con lo que sabíamos del proyecto y observando esta situación, no podíamos más que admirarnos de la labor de los funcionarios. El valor de los instrumentos de la etnografía fue incalculables para entender el proceso y para la observación.

Sin embargo, esta transformación fue también dolorosa en varios aspectos: las condiciones laborales cambiaron, la gestión se volvió la del Estado y la URT empezó a ser el blanco de intrigas políticas, de duros cuestionamientos y excesivo control de actores estatales y no estatales. Ya en 2014, con la URT andando, los funcionarios a nivel nacional se quejaban de

2 Para más detalle ver Rodríguez y Estrada (2014), Rodríguez y Estrada (2015). 
trabajar más para responder a las instituciones y a los observatorios, que para adelantar la política.

Este proceso de cambio merece una reflexión más amplia pues tiene la relevancia de mostrar el cambio de las lógicas de gobierno en torno a la política de protección patrimonial, la riqueza de ver al Estado en plena trasformación y confirma el análisis sobre la superposición de poderes en la puesta en marcha de formas de gubernamentalidad.

\section{Implicación e Intervención en la Política de Restitución de Tierras.}

Una discusión que parece relevante resaltar tiene que ver con los posibles campos de interacción entre la Investigación académica y la intervención social, trabajado en otros documentos (Rodríguez 2015). Si bien el objetivo de esta investigación en un principio fue académico, la coyuntura llevó a un involucramiento de los investigadores en consultorías y en la propia URT. Este es un caso interesante, aunque no aislado, de las dinámicas en que hoy en día se mueve la investigación antropológica y por eso quiero desarrollar una última reflexión en torno a este aspecto.

En primer lugar, es de resaltar que el trabajo etnográfico fue un espacio privilegiado para comprender la problemática de la protección y la restitución; a la vez que se analizaban los documentos jurídicos y técnicos, fuimos comprendiendo todo el proceso de materializar y poner en marcha la política de protección. Esto nos permitió tener acceso a información privilegiada sobre lo que había pasado en relación a la protección de la tierra, pero sobre todo sobre lo que estaba pasando en relación a la formulación y puesta en marcha de la política de restitución. Paralelamente, las entrevistas con los funcionarios se iban convirtiendo en cátedras de derecho civil, agrario, de ingenia catastral y de trabajo con la población desplazada. Se podría decir que se produjo un proceso de transmisión de conocimientos sobre el tema, de la mano de quienes gestaban y gestionaban los avances en todos esos aspectos.

En segundo lugar, al abordar un tema de coyuntura política, se nos permitió ver en funcionamiento los espacios y estrategias políticas en juego para poner en marcha la restitución. Esta perspectiva de observador se convirtió también en un rol privilegiado para entender tanto los beneficios, como las críticas que empezaban a surgir sobre el proceso. Como científicos sociales, creemos que esta comprensión de las arenas políticas puede dar un valor agregado cuando nos involucramos en procesos de intervención.

Por otra parte, la visión reflexiva desarrollada a partir de la perspectiva etnográfica nos permitió también posicionarnos respecto al tema; identificar fortalezas, pero también debilidades, conocer las controversias entre los distintos funcionarios dentro y fuera del proyecto y obtener los análisis de la coyuntura por parte de funcionarios que ya habían salido. Toda esta información y diversidad de perspectivas, analizadas desde un punto de vista más "distanciado o reflexivo" en relación a la mirada de quienes estaban al pie del cañón en la defensa de la política, favoreció el hecho de que al involucrarnos en un proceso de investigación acción como consultores; pudiésemos hacer aportes relevantes y propuestas de mejoramiento o fortalecimiento. 
Finalmente, pero no menos importante, las relaciones de confianza que logramos establecer con los funcionarios durante el desarrollo de la etnografía ayudaron a que fueran conociendo nuestro trabajo, que fueran valorando nuestras opiniones y que conocieran nuestras intenciones y características personales. Desde un sentido práctico, el haber establecido estas relaciones, fue sin duda un punto a favor para luego podernos involucrar de manera más activa.

Como resultado de esta faceta más implicada el proceso de aprendizaje se completó al habernos involucrado y nos permitió ver otras facetas del ejercicio político que también merecen ser analizadas en detalle.

Para terminar, quisiera solamente mencionar el hecho de que el trabajo de consultoría permitió ver también las dificultades en la implementación de la Ley, entender que no basta solo con voluntad política y que desafortunadamente ni el país ni las instituciones estaban preparadas para atender la magnitud de lo que fue el despojo. Por otra parte, a pesar de la gran inversión de recursos, los resultados no son los esperados y las intervenciones a través de consultorías, al ser cortas, sin continuidad, y muchas veces sin mucho compromiso por parte de las empresas que las gestionan y las agencias que las financian; lamentablemente no tienen el impacto que deberían tener en favorecer los procesos de implementación de la política de restitución. En cuanto a la experiencia haciendo parte del Estado, se observó que durante la transformación del PPTP se perdió gran parte del impulso que hizo particular la incidencia de los funcionarios, en un momento de esta historia. Hoy en día la situación ha cambiado. Sin demeritar los avances producidos en esta materia, es de señalar que diferentes circunstancias políticas y sociales limitan aun el proceso y no auguran todos los buenos resultados que se esperaban con la restitución.

\section{Bibliografía}

Abrams, P. (1988). "Sobre la dificultad de estudiar al estado". Blackwell Publishing Ltd. pp $112-131$.

CODHES - UNICEF. (1999) "Un país que huye. Desplazamiento y violencia en una nación fragmentada". Bogotá.

Fassin, D (2009): Les sciences sociales critiques peuvent-elles être útiles? Ciclo de conferencias: "A quoi servent les sciences humanes". Revista Tracés: http://www.dailymotion.com/video/x9pygm_une-science-sociale-critique-peutel_news. Fecha de publicación 26-06-2009. Fecha de consulta 06-2012

Ferguson, J (1994) the Anti-Politics Machine: “'Development,' De politicization, and Bureaucratic Power in Lesotho. Minneapolis: Universtity of Minnesota Press.

Haidar, V (2011). El ‘descentramiento’ del Estado en el análisis del poder (político): Un diálogo crítico entre la sociología histórica y el enfoque de la gubernamentalidad. Espacio Abierto. [Online]. Abr. 2005, vol.14, no.2

Ibáñez, Ana María y Vélez, Carlos Eduardo (2003). Forced displacement in Colombia: causality and welfare loses. Universidad de los Andes y Banco Mundial.

Nugent, D (1997) Modernity at the Edge of Empire: State, Individual, and Nation in the Northern Peruvian Andes, 1885-1935. Stanford, CA: Stanford Universtity Press. 
Rodríguez, N (2015). Características y tipologías de la investigación en ciencias sociales: una reflexión sobre la complementariedad de las funciones de transformar y comprender en: Revista Universitas Humanística. Universidad Javeriana ISSN: 01204807 No 81 2015. (Artículo aprobado en Edición)

Rodríguez, N y Estrada R (2015). Tácticas de gobiernos en la política de protección de tierras en Colombia: Una Etnografía del Estado. (Manuscrito en Evaluación).

Rodríguez, N y Estrada, R (2014). La política de tierras para la población desplazada 2001-2011: de la protección a la restitución. Revista Estudios Socio Jurídicos. Universidad del Rosario ISSN: 0124-0579. Vol. 16. N1. pp75 a 119.

Rose, N \& Miller P. (1992). Political Power beyond the State: Problematics of Government. The British Journal of Sociology, vol. 43, 2.

Sarmiento, Libardo (2000). "Desarrollo societal, conflicto y territorio". Ponencia presentada al Seminario Internacional "Desplazamiento, conflicto, paz y desarrollo", mayo 30 a junio 2 de 2000. Bogotá: Codhes

Scott, J (1998). Seeing Like a State: How Certain Schemes to Improve the Human Condition. Have Failed. New Haven, CT: Yale University Press.

Sharma, A., \& Gupta, A. (Eds.). (2006). the anthropology of the state: a reader. John Wiley \& Sons.

Swartz, M; V W, Turner. A, Tuden. 1996. “Introduction”. En Political Anthropology. Chicago: Aldine Press. (Págs $102-109$ )

Trouillot, M-R (2011). Transformaciones globales: La antropología y el mundo moderno. Editorial Universidad Del Cauca.

(2003).the Anthropology of the State in the Age of Globalization: Close Encounters of the Deceptive Kind. In Global Transformations: Anthropology and the Modern World. Pp. 79-96. New York: Palgrave Macmillan. /

(2001). the Anthropology of the State in the Age of Globalization: Close Encounters of the Deceptive. Current Anthropology, Vol. 42, No. 1 (February 2001), pp. 125-138. The University of Chicago Press.

Uprimny, R., \& Sanín, M. P. S. (2006). Justicia transicional y justicia restaurativa: tensiones y complementariedades. R. Uprimny Yepes, Justicia transicional sin transición: reflexiones sobre la verdad, justicia y reparación en Colombia. Bogotá: De Justicia.

Villa, M (2006). "Desplazamiento forzado en Colombia. El miedo: un eje transversal del éxodo y de la lucha por la ciudadanía". Controversia, noviembre 2006. 